- The use of disposable mercury monitors in dental surgeries throughout the United Kingdom suggests that over the past 20 years mercury hygiene practices by members of the dental team have improved.

- Disposable mercury monitors are useful as first-level indicators of hazardous concentrations of mercury vapour in dental surgeries.

- Whilst the use of encapsulated amalgam eliminates the need for handling mercury, the careful use of well-maintained amalgamators provides a safe alternative.

\title{
Twenty years of mercury monitoring in dental surgeries
}

\author{
D. Brown ${ }^{1}$ and M. Sherriff ${ }^{2}$
}

From 1981 to 2001 disposable mercury monitors based on palladium chloride have been deployed in 3,568 dental surgeries throughout the United Kingdom. They have performed well as first-level indicators of high concentrations of mercury vapour in surgeries that had become contaminated by spillage of mercury or faulty equipment. Whilst accumulated data suggests that mercury hygiene practices have improved over the past 20 years, the move to the use of disposable capsules rather than automatic amalgamators does not appear to have been a major factor in this improvement.

Ever since its early misuse by rogue operators in the USA in the 1830s, dental amalgam has always been under suspicion because it is based on mercury. During the 20th century much data has been accumulated on the toxicology of mercury, and analytical equipment has become so sensitive that it is now possible to detect small quantities of mercury in any body tissue or fluid, and even in expired air from the mouths of those with amalgam fillings. Thus, when a patient has never been exposed to other sources of mercury (such as those of industrial, pharmacological or dietary origin) it is possible to relate their body burden of mercury to the presence of amalgam fillings. ${ }^{1}$ Using analytical equipment, researchers have been able to detect mercury in the kidneys of sheep with amalgam fillings. However, even in animals with such exaggerated masticatory habits as theirs, the mercury derived from the amalgam fillings produced no clinical manifestations ${ }^{2}$.

${ }^{1 *}$ Senior Lecturer, Dental Biomaterials Science Department, GKT Dental Institute at Guy's Hospital, London SE1 9RT; ${ }^{2}$ Senior Lecturer, Dental Biomaterials Science Department, GKT Dental Institute at Guy's Hospital, London SE1 9RT

Correspondence to: D. Brown

E-mail:david.2.brown@kcl.ac.uk

\section{Refereed Paper}

Received 02.11.00; Accepted 06.09.01

๑) British Dental Journal 2002; 192: 437-441
The American Council on Science and Health has determined that allegations against amalgam restorations constitute one of the greatest unfounded health scares of recent times, ${ }^{3}$ and in a masterly assessment in 2001 using the paradigm of evidence-based care, Dodes supports the US Public Health Services view that "there are no data to compel a change in the current use of amalgam. ${ }^{4}$

Although alternatives to amalgam such as dental composites are now highly sophisticated materials, their handling characteristics and properties once set still limit their use as a viable alternative to dental amalgam for producing large fillings in posterior teeth. Thus whilst the USA, Canada, Australia and countries within the European Union are all undertaking risk assessments of amalgam, they are also evaluating alternatives such as composites in the same way, for despite many countries having sought to restrict the use of amalgam either by direct edict or by making its use economically unrealistic, none has decreed that amalgam should never be used.

On the other hand, it is well documented that when elemental mercury is misused or handled without care in any industry or profession, those exposed to its vapour are at considerable risk. Although a droplet of pure mercury will produce a concentration of vapour determined by the surrounding air pressure and temperature, its surface soon becomes oxidised and the quantity of vapour emitted diminishes. However, a slight disturbance will break this barrier and release the vapour. Raising the temperature produces a rapid rise in the vapour pressure and this is enough to break the thin oxide barrier and release it. Hence, allowing mercury or set amalgam to get hot can create hazardous concentrations of vapour within an unventilated space.

An extreme example of this was reported in 1990, when four residents in a Detroit apartment block perished from acute mercury intoxication within a few days of being exposed to high concentrations of mercury vapour. ${ }^{5}$ This was generated by trying to recover silver from dental amalgam by smelting it in a furnace in the basement of the house.

In 1976 an instance of chronic mercury intoxication was reported from a dental practice in Surrey, when four members of a dental team were exposed over a period of 3 months, during the winter months when ventilation was reduced, to vapour generated from a hidden, heated mercury spillage. ${ }^{6}$ Although between them the four individuals showed many of the classic signs and symptoms of chronic hydragyrism, once they had been removed from their surgeries and treated with 
chelating agents to flush the mercury out of their bodies disaster was averted. However, in 1969 a dental surgery assistant (dental nurse) was not so fortunate when she succumbed to chronic mercury intoxication from an unknown and undetected source in the surgery in which she had worked for 20 years. ${ }^{7}$ In her case the intoxication led to the development of a nephrotic syndrome, which proved to be irreversible and she died.

In these latter two cases, the use of some form of mercury monitor could have warned them of their chronic exposure to high concentrations of mercury vapour.

\section{MERCURY MONITORS}

Two types of monitoring can be undertaken in a dental surgery - active and passive.

Active monitors employ chemical analytical techniques to instantly assess the amount of mercury present in the air. For example, one type draws air into a tube continuously by means of a pump. At one end of the tube is a source of ultra-violet (UV) light and at the other a UV detector. Mercury absorbs UV light and an electronic circuit compares the electrical output from both source and detector. A digital read-out shows the concentration of mercury in the air. These devices cost several thousand pounds, and whilst this makes them prohibitive for routine use in the dental surgery, they can be used by agencies seeking sources of mercury vapour, which have been suggested as a result of passive monitoring.

Passive monitors, as their name suggests, just sit there and absorb any mercury that happens to be in the air of the surgery. Those who work in the dental surgery make very fine passive monitors, and by analysing any body fluid or tissue that can be collected, it is possible to assess the amount of mercury to which their donor has been exposed. In the past, blood, urine, hair and nail have all been analysed by complex techniques using sophisticated equipment. An alternative is to expose a material that undergoes some sort of physical or chemical change when mercury comes into contact with it. During the 20th century adsorption of mercury by gold and its absorption into charcoal were each employed as passive mercury monitors. The gold underwent a change in its electrical conductivity, which could be measured, and after it had been exposed, the charcoal was encouraged to liberate its mercury burden inside an analytical chamber. However, a simpler form of passive monitor uses one of a number of time and concentration dependent chemical reactions that result in a colour change. The chemicals can be held on absorbent paper or contained in glass tubes. In the latter form, a known volume of air is drawn through the tube via a pump, whereas the papers are exposed in the air of a room for several hours or days.

Yellow palladium chloride is reduced by mercury vapour to metallic palladium. As it is finely divided the palladium appears dark, and in 1974 Jørgensen suggested that paper discs that had been soaked in a $1 \%$ solution of palladium chloride and allowed to dry could be used to monitor the presence of mercury in dental surgeries. ${ }^{8}$

This became the basis for the "Williams Detector' marketed in the 1970s and 80s by the Williams Gold Refining Co. Inc of Buffalo, USA. It consisted of a filter paper that had been soaked in palladium chloride, dried and mounted on a card for hanging in the dental surgery. The card bore the information that 'This palladium chloride treated filter paper will turn gray if MERCURY VAPOR is present'. However, as the reaction is dependent on both time and concentration, long exposures to very low concentrations of vapour will eventually turn the disc grey. Thus the discs supplied to dental surgeries in the study to be described were exposed for a standardised time of 7 days.

In 1983 Brown reported the results of using such passive monitoring discs in 704 dental surgeries throughout the United Kingdom. ${ }^{9}$ This account extends that study up to August 2001. During the period March 1981 to August 2001 some 3,568 surgeries, in which amalgam was being triturated and used as a filling material, have been assessed.

\section{METHODS AND MATERIALS}

Monitoring discs were made by impregnating $3 \mathrm{~mm}$ diameter circles of Whatman's No 1 filter paper with a $1 \%$ solution of palladium chloride. The solution was prepared by dissolving $1 \mathrm{~g}$ of the brown salt $\mathrm{Pd}(\mathrm{II}) \mathrm{Cl}_{2}$ in $100 \mathrm{ml}$ of deionised water, to which had been added $5 \mathrm{ml}$ of concentrated hydrochloric acid. After filtration N/10 potassium hydroxide was added to the stirred solution until it reached $\mathrm{pH}$ 5. The filter paper discs were soaked in batches of 15 with this solution, squeezed to remove excess and dried on sheets of absorbent paper at $60^{\circ} \mathrm{C}$. The paper sheets were ultimately ashed in order to recover the palladium.

\section{Distribution}

In the early 1980s the discs were given out during clinical meetings at which the authors were speaking, and in 1982 advertisements in the dental press produced many requests for the offer of a free mercury monitoring service.

In 1988, the Associated Chemical Company, then based in the West End of London, agreed to sponsor this investigation into the viability of these discs for monitoring dental practices. Thus they offered the discs and their subsequent assessment as a free service to those dental practitioners they hoped might purchase their amalgam alloy.

In 1991, the Schein Rexodent Company of Southall put the discs on a commercial footing by giving them away for nothing but charging a fee for their assessment. The authors' Department carried out this assessment in return for a proportion of the fee. As the Company expanded to become Henry Schein Rexodent, then Henry Schein Procare and now Henry Schein of Gillingham, Kent this service has continued.

Soluble glue based on polyvinyl pyrrolidone with some polyvinyl alcohol components (Pritt Stick) was used to attach each disc to a piece of card. This was sent to the practitioner together with a form bearing instructions for use. These included the suggestion that the disc should be mounted in the dental surgery at about 'working head height' and left in position continuously for 7 days. The form also sought information on how amalgam was mixed in that surgery, what type of ventilation was in use and what the average temperature had been.

\section{Assessment}

On receipt, each disc was given an identification number and this was marked on the disc with a pencil. It was then immersed in a 50\% solution of ammonium hydroxide, with the result that two things happened. The glue softened so that the disc could be detached from the card and any yellow palladium chloride that had not been reduced to grey metallic palladium in the reaction:

$$
\mathrm{PdCl}_{2}+\mathrm{Hg} \rightarrow \mathrm{Pd}+\mathrm{HgCl}_{2}
$$

was converted into a colourless palladiumammonium-hydroxy-complex, probably by the reaction:

$\mathrm{PdCl}_{2}+\mathrm{NH}_{4} \mathrm{OH} \rightarrow \mathrm{Pd}\left(\mathrm{NH}_{3}\right)_{4}(\mathrm{OH})_{2}+\mathrm{NH}_{4} \mathrm{Cl}$

The discs were dried on absorbent paper in air at $60^{\circ} \mathrm{C}$ prior to being placed in the light beam of a simple photometer, where its transmission was compared with that of an unexposed disc. A percentage transmission value was the result.

\section{Significance of the transmission value} In 1983, the threshold limit value (TLV) for mercury was 50 micrograms per cubic metre. The TLV of a substance is a level set by safety authorities throughout the world. It is said to represent that concentration of a substance to which those regularly exposed can be exposed throughout their working lives without being in danger of suffering adverse effects to their health. It 
assumes they are working 8 hours a day, 5 days a week. In 2001 the TLV is also referred to as the OEL (the occupational exposure limit). This limiting value is known as the Long-Term Limit in the UK and as the Limit Value (TWA) in the USA. In both countries its value is now 25 micrograms per cubic metre of air.

In 1983 Brown described the use of a photometer to determine the response of these discs to the concentration of mercury in air and hence to decide which surgeries were operating at or above the TLV of 50 micrograms per cubic metre. The photometer was calibrated by using monitoring discs that had been exposed in a room that was deliberately contaminated with various concentrations of mercury vapour for a week.

This enabled a plot to be made of per cent transmission of the disc against the average concentration of mercury in the room. In 1983 discs with transmission values below 55\% indicated that the surgeries from which they came contained air with mercury vapour concentrations that were above the TLV of 50 micrograms per cubic metre. The reduction in 1996 of the limit to 25 micrograms per cubic metre meant that those surgeries returning discs that proved to have transmission values below $70 \%$ received special attention. Such surgeries received two repeat discs together with the request that one should be placed at working head height and the other 2 inches above the floor and that both should be exposed for 7 days. If the repeat discs indicated that mercury was present industrial mercury monitors were supplied to the practice.

All who sent discs for assessment received a written report, advice on the handling of mercury in the dental surgery and a disposable plastic pipette to enable spillages, whether small droplets or big pools, to be collected for transfer to the surgery's waste amalgam container.

\section{RESULTS}

\section{First and second assessments}

Since 1981, reports have been sent to 3,568 surgeries following the first assessment of discs exposed at head height. Four hundred and sixty-seven of these surgeries were invited to repeat the assessment because the transmission values of their discs fell below 70\%. Except for a few, the second assessments resulted in mean values of $84 \%$ for the head height disc and $82 \%$ for the one that was positioned close to the floor. During the period 1981 to 1985 14\% of surgeries required repeat discs; from 1986 to 1990 this fell to 8\%; during the next five years 9\% required a second assessment and from 1996 to 2001 this dropped to $7 \%$. The total of 3,568 includes

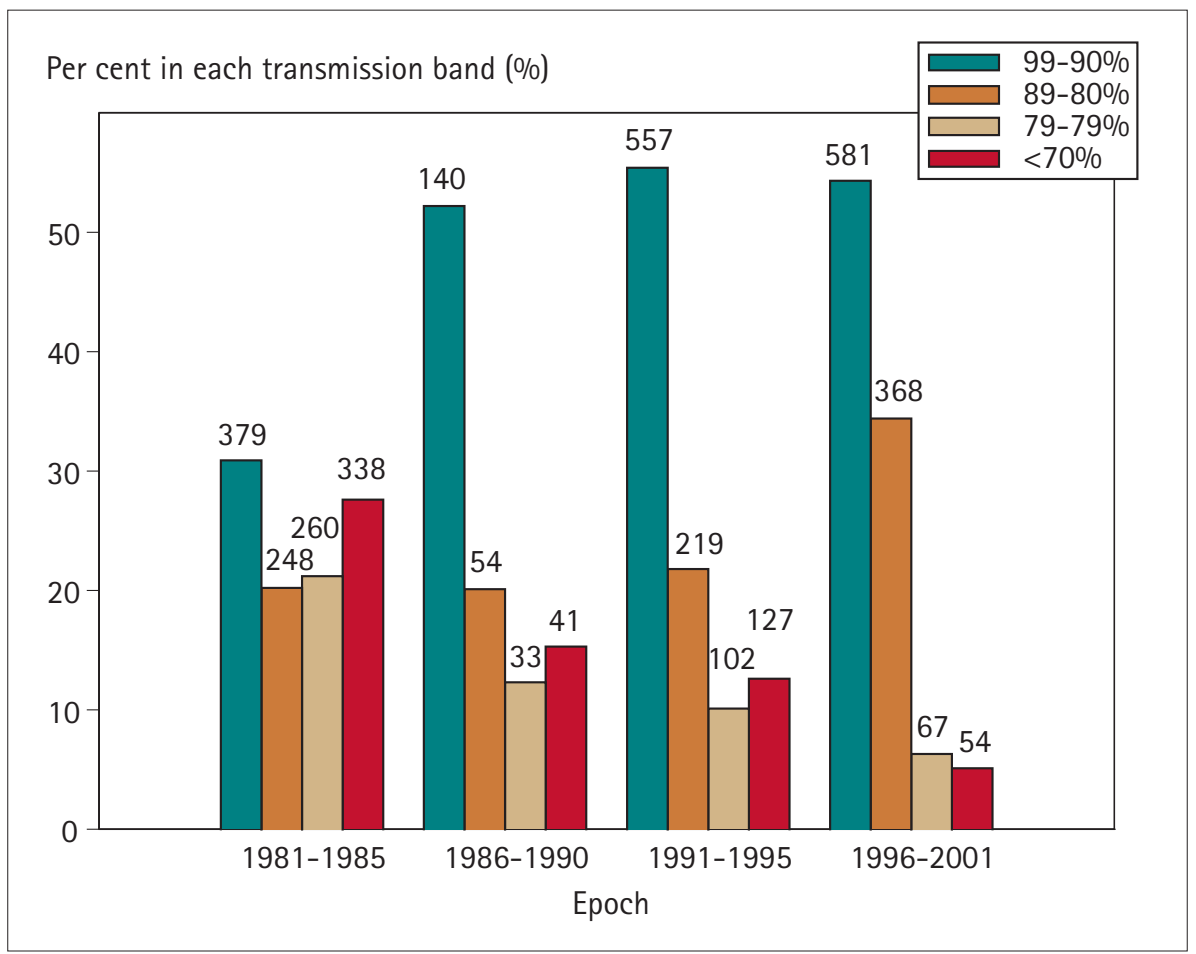

Fig. 1 The per cent transmission of 3,568 mercury monitoring discs assessed between 1981 and 2001

143 surgeries that have undertaken a first assessment on more than one occasion.

\section{Quinquennial transmission values}

Figure 1 shows the distribution of light transmitted by exposed discs during the four periods of 5 years since 1981. Four divisions are shown - green, orange, gold and red. Green represents the transmission values between 99 and 90\%, orange transmission values between 89 and $80 \%$, gold values between 79 and 70\% and red tranmission values below 70\%.

Low transmission values indicate that the discs had been exposed to a significant presence of mercury vapour, whereas high transmission values indicate exposure to low concentrations. In this figure the percentage of discs showing high transmission values increases from 1981 to 2001, whilst those showing lower values decreases during the same period.

\section{Mixing techniques}

During the period 1981 to 1990 practitioners were requested to indicate whether they mixed their amalgam by hand, using a re-fillable capsule, in a disposable capsule or in one of several automatic amalgamators, including the 'Amalgamiser' and the 'Dentomat'.

Whilst hand-mixing was being used by $16 \%$ of the practices sending back discs in 1981 , by 1986 it had fallen to less than $1 \%$, and after this date no practices were triturating amalgam by hand.

By 1990 it became clear that those surveyed used only either a pre-proportioned, disposable capsule or a Dentomat to tritu- rate their amalgam, and since then, as shown in Figure 2, there has been a steady rise in the percentage of practices using disposable capsules.

Between 1994 and 2001 some 895 discs were assessed from surgeries that supplied information about which of the two contemporary methods was being used to prepare the dental amalgam. Figure 3 compares the percentages of surgeries in each of four ranges of transmission for amalgam mixed either in a disposable capsule or in a Dentomat. The symmetry of this figure suggests that there is no relationship between the method of preparation and the mercury vapour detected.

\section{Practice environment}

The forms that accompanied each disc provided information about the practices using these discs. They showed, for example, that in the quinquennium 1981 to 1985 the average number of surgeries at a particular address was 1.4, but in the period 1996 to 2001 this had risen to 1.9 .

Figure 4 indicates the percentage of surgeries where discs were deployed that had extractor fans to assist in the circulation of air. It also shows a gentle increase in the use of air conditioning over the past 20 years.

Practices were asked to indicate the temperature in the surgery in which the disc was placed. Of those that did, Figure 5 shows how the percentage of warm surgeries, ie those with air temperatures between 70 and $70^{\circ} \mathrm{F}\left(21-25^{\circ} \mathrm{C}\right)$ increased slightly over the past 20 years. The cool range was taken to be 60 to $65^{\circ} \mathrm{F}\left(15-20^{\circ} \mathrm{C}\right)$. 


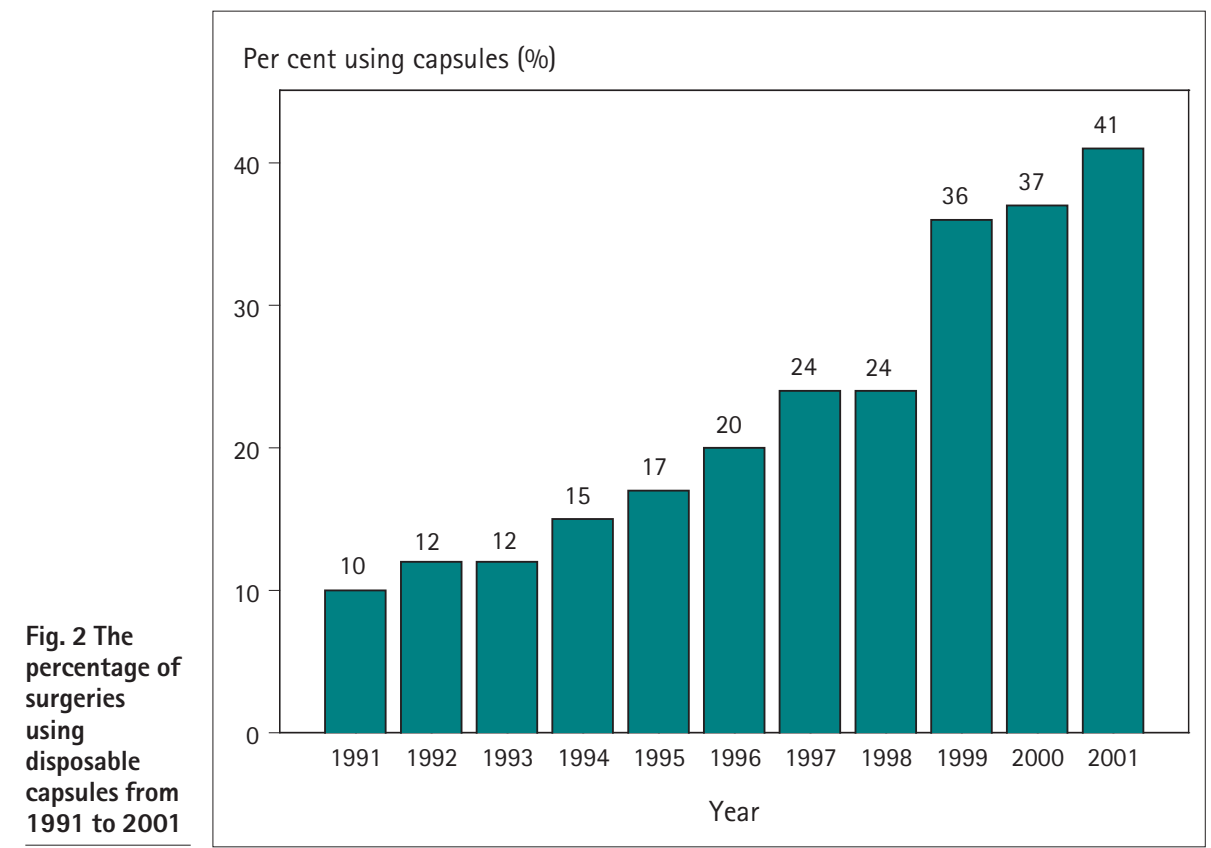

\section{DISCUSSION}

Analysis of the assessments suggests that mercury hygiene practices by members of the dental team have improved over the past twenty years. This conclusion is based on two observations.

Firstly, in the period 1995-2001 when single monitoring discs were exposed for 7 days in the atmosphere of many surgeries, fewer indicated a significant presence of mercury, and thus required repeat assessments than were needed in the period 1981-95.

Secondly, over the four quinquennia, as is shown in Figure 1, there is an increase in the percentage of assessments at the green and orange levels (indicative of low mercury exposure) and a decrease in those at the yellow and red levels (indicative of higher exposure to atmospheric mercury).

To what might this improvement be attributed? Perhaps the general reduction in the number of amalgam restorations being placed in the United Kingdom means that less mercury is being involved. However, all the discs returned for analysis came from surgeries in which amalgam was being handled.

The disappearance of the hand mixing of amalgam from the dental surgeries of the United Kingdom is one contributory factor, as might be the move away from refillable automatic amalgamators such as the Dentomat towards disposable, pre-proportioned capsules, as recorded in Figure 2. However, Figure 3 shows that there was no real difference in the distribution of transmission values in the green, orange, yellow and red divisions when some 883 discs assessed over the past 7 years were considered with respect to whether amalgamation took place in a Dentomat or in a disposable capsule.
Those surgeries that were invited to undertake repeat assessments because the transmission values of their first discs were less than 70\% (or less than 55\% from 1981 to 1995) came up with mean transmission values of $84 \%$ for the discs exposed at head height and 82\% for those exposed 2 inches above the floor. This suggests that the warning had either encouraged an improvement in mercury hygiene practices, or the members of the dental team had sought out and removed a hidden spillage or other persistent source within the surgery.
Per cent of discs (\%) capsules from 1994 to 2001
In some cases even the repeat discs suggested that there were hidden sources of mercury vapour in the surgeries in which they had been exposed, and the authors investigated several of these using an active monitor. It became clear that spillage associated with attempts to fill the mercury chamber of automatic amalgamators was a frequent cause of contamination. In two surgeries, mercury had been spilled onto carpeted surfaces, and in two others the amalgamators had developed cracks in their mercury reservoirs. Examination showed that these cracks only opened during trituration, and when they did mercury was observed weeping from them. A recent report on the status of 11 amalgamators belonging to the USAF brought to light similar leakages, although they were found to make only a minor contribution to the mercury vapour present in the dental surgeries in which they were used. ${ }^{10}$ Figure 3 suggests that when these devices were filled carefully, maintained regularly and serviced occasionally their contribution to mercury hygiene in the dental surgery has been a valuable step forward. Whilst disposable capsules are a viable alternative, they do present the added challenge of the safe disposal of the used capsule.

On several occasions, contaminated aspirators proved to be the source of vapour, thus confirming the recent ${ }^{11}$ and earlier ${ }^{12}$ reports. These particular devices vented into the surgery rather than outside the building. The crenellations in the

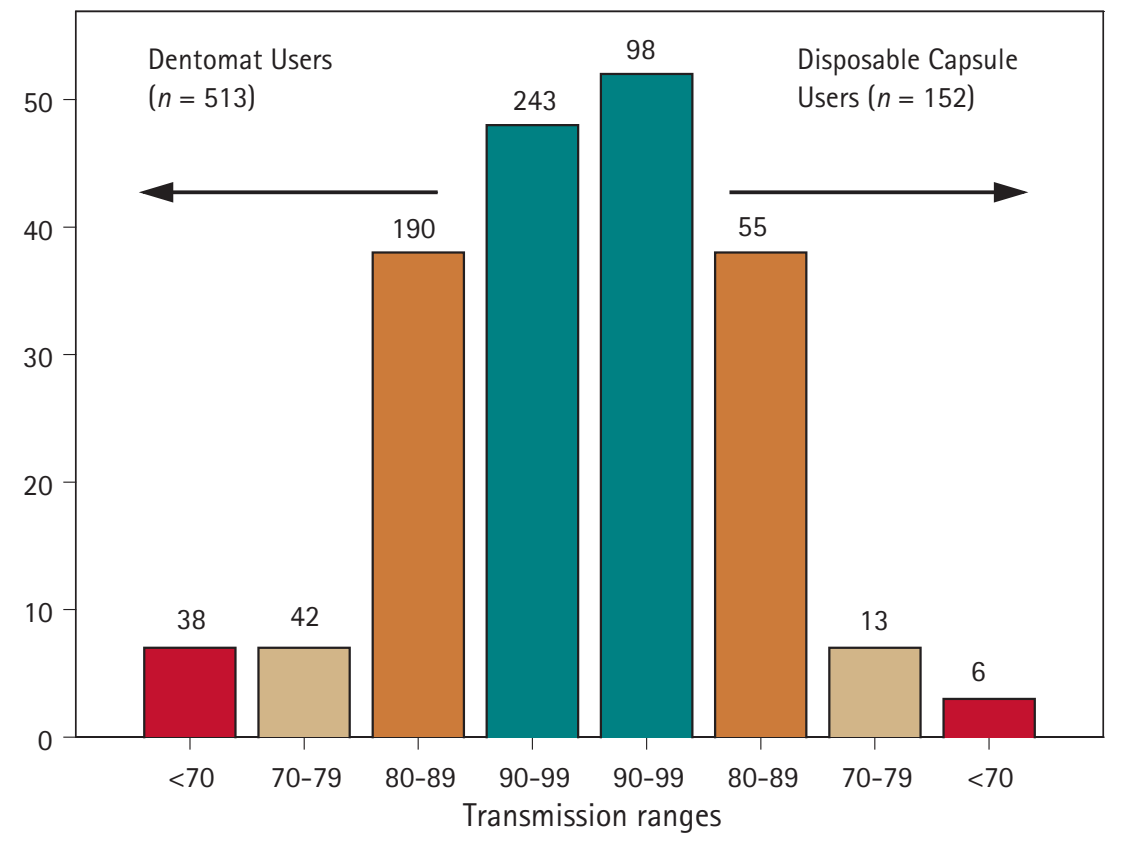

Fig. 3 Comparison of the per cent transmission values from users of either Dentomats or disposable 

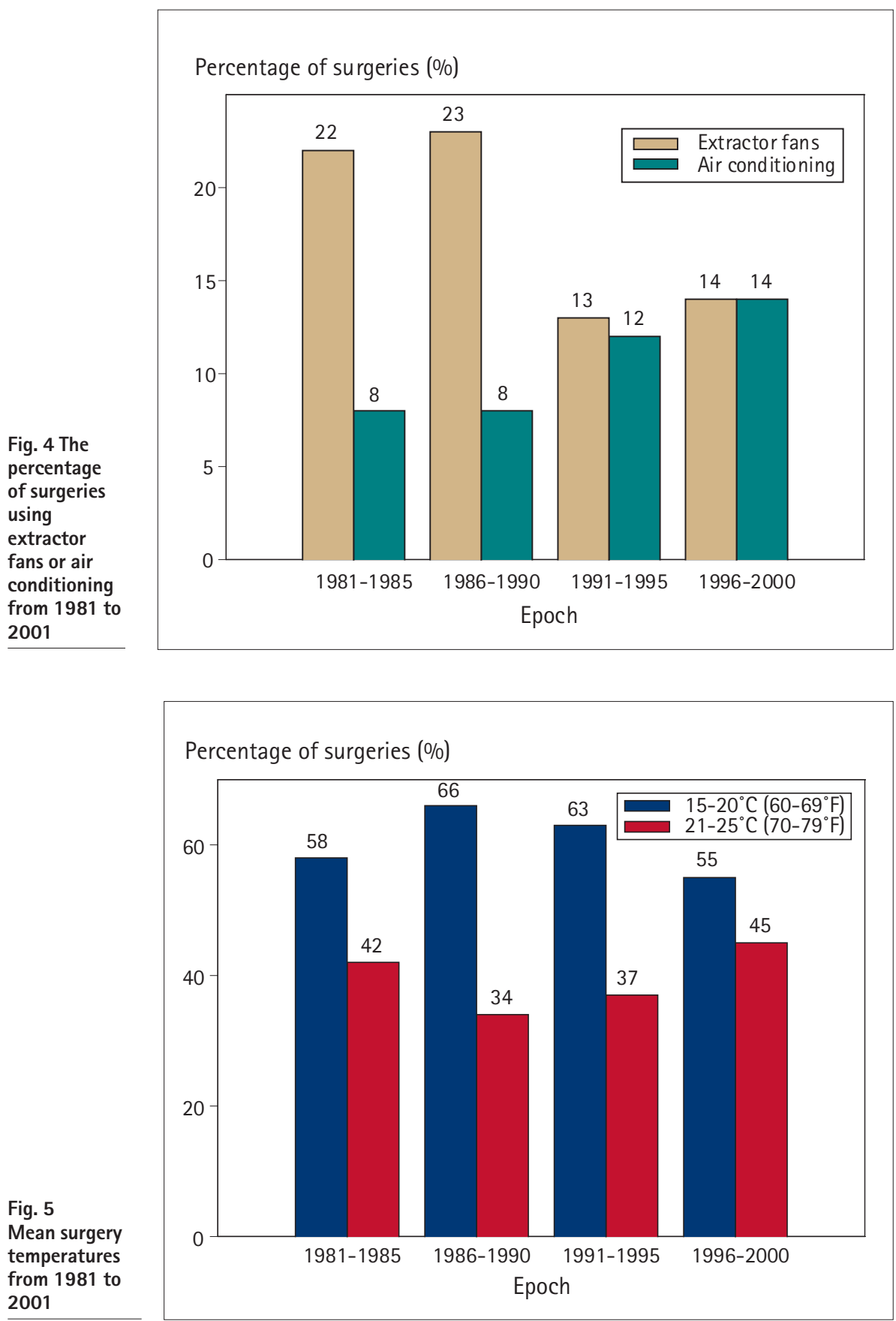

flexible tubing and the filters in this sort of equipment require regular cleaning to keep such contamination under control.

From the information provided by those practices that had chosen to use the mercury monitoring discs it has been of interest to note the increase in the mean number of surgeries at each practice address, the slow acceptance of air conditioning (which, incidentally tends to distribute the vapour from mercury spillages in one surgery around the rest of the

\section{CONCLUSIONS}

Over the past 20 years these disposable mercury-monitoring discs have proved to be reliable, first-level indicators of high concentrations of mercury vapour in contaminated dental surgeries. The accumulated data from the assessment of 3,568 discs suggests that:
- There was an improvement in mercury hygiene practices in dental surgeries during the period 1981 to 2001

- When automatic amalgamators are handled carefully and maintained regularly they make the same valuable contribution to mercury hygiene as do disposable capsules

- Hand-mixing of amalgam in the United Kingdom is a thing of the past,

- The careless handling of mercury over carpeted floors can contribute to high concentrations of atmospheric mercury vapour

- Since 1981 , the number of surgeries at each practice address has increased, practices are slightly warmer and more have air conditioning.

The authors wish to acknowledge Henry Schein and the Associated Chemical Company for their financial support during the trial of these devices.

Suppliers of materials, equipment or services: Amalgamiser - Amalgamated Dental Co (now part of Dentsply Ltd, Weybridge, KT15 2BR)

Associated Chemical Company Northolt, UB5 5AY

Dentomat - Degussa Ltd, Handforth, SK9 3RL

Henry Schein ${ }^{\circledR}-$ Gillingham, Kent ME8 OSB Pritt Stick - Henkel Consumer Adhesives, Winsford, CW7 3QY

Whatman Filter Paper - Whatman International Ltd, Maidstone, ME16 OLS

Palladium(II) Chloride - Merck Ltd, Poole, BH15 1TD

1. Eley B M. The future of dental amalgam: a review of the literature. Part 5: Mercury in urine, blood and body organs from amalgam fillings. Br Dent J 1997; 182: 413-417.

2. Boyd N D, Benediktsson H, Vimy M J etal. Mercury from dental 'silver' tooth fillings impairs sheep kidney function. Am J Physiol 1991; 261: R1010-1014.

3. Lieberman A J, Kwon S C. Facts versus fears: a review of the greatest unfounded health scares of recent times. 3rd ed. New York: American Council on Science and Health, 1998.

4. Dodes J E. The amalgam controversy: an evidencebased analysis. J Am Dent Assoc 2001; 132: 348-356.

5. Kanluen S, Gottlieb C A. A clinical pathologic study of four adult cases of acute mercury inhalation toxicity. Arch Pathol Lab Med 1991; 115: 56-60.

6. Merfield D P, Taylor A, Gemmell D M, Parrish J A. Mercury intoxication in a dental surgery following unreported spillage. Br Dent J 1976; 141: 179-186.

7. Cook TA, Yates P O. Fatal mercury intoxication in a dental surgery assistant. Br Dent J 1969; 127: 553-555.

8. Jørgensen K D. A semi-quantitative test for mercury in air. Acta Odont Scand 1974; 32: 305-308.

9. Brown D. Detection of mercury vapour in the dental surgery. Br Dent J 1983; 155: 237-240.
10. Roberts H W, Leonard D, Osborne J. Potential health and environmental issues of mercury-contaminated amalgamators. J Am Dent Assoc 2001; 132: 58-64.

11. Stonehouse CA, Newman A P. Mercury vapour release from a dental aspirator. BrDent J 2001; 190: 558-560.

12 Smart ER. Mercury vapour from aspirators. Br DentJ 1990; 168: 138 (letter) 